\title{
Evaluation de la compétitivité des systèmes piscicoles pratiqués en Côte d'Ivoire: gestion, alimentation et production
}

\author{
Hortense A. YAO ${ }^{1,2}$, Rachel A. KOUMI ${ }^{2 *}$, Céline Sidonie K. NOBAH ${ }^{3}$, \\ Célestin B. ATSE ${ }^{2}$ et Paul E. KOUAMELAN ${ }^{1}$ \\ ${ }^{1}$ UFR Biosciences, Université Félix Houphouët-Boigny, 22 BP 582 Abidjan 22, Côte Abidjan, Côte d'Ivoire. \\ ${ }^{2}$ Centre de Recherches Océanologiques, BP V 18, Abidjan, Côte d'Ivoire. \\ ${ }^{3}$ Ecole Normale Supérieure d'Abidjan (ENS), 08 BP 10 Abidjan 08, Côte d'Ivoire. \\ *Auteur correspondant ; E-mail : koumirachel@yahoo.fr ; BP V 18, Abidjan, Côte d'Ivoire. \\ Tel : +22507871069
}

\section{RESUME}

La production aquacole de poisson reste faible en Côte d'Ivoire. Pour évaluer la capacité des systèmes pratiqués à produire des poissons marchands compétitifs en quantité suffisante, les indicateurs de gestion, d'alimentation et de productivité des systèmes piscicoles ont été évalués sur 301 fermes. Les résultats permettent d'observer que les systèmes semi intensif $(51,83 \%)$ et extensif $(42,86 \%)$ étaient les plus pratiqués par rapport à la rizipisciculture (3,99\%) et à l'intensif (1,33\%). Les fermes enregistrées en intensif respectent les indicateurs de gestion évalués et enregistrent 50 à $75 \%$ de pratique de la majorité des indicateurs d'alimentation. L'intensif contribue à la production de poissons marchands compétitifs avec un rendement de plus de $10000 \mathrm{~kg} / \mathrm{ha} / \mathrm{an}$ sur toutes les fermes. Le système semi intensif traduit le suivi de la majorité des indicateurs de gestion par plus de 50\% des fermes contrairement aux indicateurs de bonnes pratiques d'alimentation et de production. L'extensif et la rizipisciculture sont caractérisés par un faible niveau de gestion, d'alimentation et de productivité. Les capacités de production en semi intensif, extensif et en rizipisciculture doivent être renforcées par les bonnes pratiques de gestion et d'alimentation. Aussi le système intensif doit être promu pour améliorer la production quantitative de poisson compétitif.

(C) 2016 International Formulae Group. All rights reserved.

Mots clés : Pisciculture, système, indicateur, gestion, alimentation, rendement.

\section{Assessment of Côte d'Ivoire fish farming systems competitivity: management, feeding and production}

\begin{abstract}
Aquaculture fish production remains low in Côte d'Ivoire. To assess the ability of fish farming systems practised to produce competitive commercial fish in quantity, the indicators of management, feeding and production associated to the fish farming systems were evaluated in 301 farms. Results showed that semiintensive $(51.83 \%)$ and extensive $(42.86 \%)$ systems were more found than intensive $(1.33 \%)$ and rice fish farm $(3.99 \%)$ systems. The fish farms in the intensive system were consistent with good management practices and fitted the majority of feeding indicators of about $50-75 \%$ of farms. Intensive system promotes competitive
\end{abstract}


commercial fish production with a yield above of $10000 \mathrm{~kg} / \mathrm{ha} / \mathrm{yr}$ in all the farms. Semi intensive system expresses the majority of management indicators by more than $50 \%$ of farms in contrast to the indicators of good feeding practices and the recommended production values. Extensive and rice fish farming systems were characterized by low level of management, feeding and production. The capacity of production in semi intensive, extensive and rice fish farming systems must be improved by enhancing the good practices of management and feeding. Moreover, intensive systems must be also promoted to increase competitive commercial fish production.

(c) 2016 International Formulae Group. All rights reserved.

Keywords: Fish farming, system, indicators, management, feeding, yield.

\section{INTRODUCTION}

Les produits halieutiques, en particulier le poisson, jouent de plus en plus un rôle essentiel dans la sécurité alimentaire, nutritionnelle et le bien être des populations des pays développés et en développement (Hossain, 2011; Toppe et al., 2012; FAO, 2013). En effet, la consommation mondiale apparente de poisson par personne est passée d'une moyenne de $9,9 \mathrm{~kg}$ au cours des années 60 à 19,2 kg en 2012 (FAO, 2014a). Avec l'épuisement des stocks halieutiques provoqués par la surexploitation des eaux, l'aquaculture est devenue depuis les années 1990, le moteur de la production de poisson destinée à la consommation (FAO, 2011). En outre, la contribution de l'aquaculture dans la satisfaction des besoins mondiaux en poisson est passée de $9 \%$ au cours des années 1980 à plus de 50\% en 2012 (FAO, 2014a). Ce progrès est spectaculaire dans certains pays d'Asie, d'Amérique latine et d'Europe mais très peu visible en Afrique, en ce sens que la part de ce continent dans la production mondiale de poisson en 2012 est estimée à seulement $2,23 \%$. L'essentiel de la production étant enregistré en Afrique du Nord avec une contribution de $1,55 \%$ contre $0,68 \%$ pour l'Afrique Sub Saharienne. La Côte d'Ivoire, avec une production annuelle de 3720 tonnes en 2012 fait partie des pays Subsahariens à faible production aquacole (FAO, 2014b). Elle enregistre une production halieutique totale de 75611 tonnes pour une consommation annuelle de 252980 tonnes (FAO, 2014b).
Pour combler ce déficit, le pays importe du poisson congelé entraînant une sortie de devise d'une valeur de 150 milliards par an (OCDE-FAO, 2011). L'augmentation de la production aquacole dans les pays en voie de développement pourrait combler le déficit évoqué, en contribuant à la création d'emploi, à la réduction de la pauvreté tout en garantissant la sécurité alimentaire et la croissance économique (Hishamunda et al., 2011).

La production aquacole est influencée par plusieurs facteurs tels que la densité de mise en charge, le taux de survie et la vitesse de croissance des poissons qui à leur tour dépendent des techniques de gestion des fermes piscicoles, de l'alimentation des poissons, des systèmes pratiqués du type de culture, du choix de l'espèce et des facteurs environnementaux (Lacroix, 2004; Harpaz, 2007 ; Toko et al., 2011, 2013 ; Elegbe et al., 2015). Par ailleurs, le choix des systèmes d'élevage de poisson varie en fonction des données géographiques et socio-économiques des producteurs (Ranjet et Kurup, 2013). De même, la production de ces systèmes est influencée par le niveau d'investissement et l'intensification de l'activité (Mungunti et al., 2014). La connaissance des systèmes pratiqués et de leur productivité constitue donc un élément important pour l'évaluation de la productivité piscicole d'un pays. Cette étude se propose d'identifier les systèmes d'exploitation piscicoles pratiqués en Côte d'Ivoire, d'en évaluer le niveau de gestion de 
production et le degré d'intensification, en vue de définir les conditions d'amélioration de la production aquacole.

\section{MATERIEL ET METHODES}

La capacité des systèmes piscicoles pratiqués en Côte d'Ivoire à produire des poissons marchands compétitifs en quantité suffisante a été évaluée selon la méthode décrite par Gobert (2008). Elle a consisté à une enquête auprès des pisciculteurs suivie de l'identification et de la classification des différents systèmes pratiqués, à la définition des objectifs et des thématiques ainsi qu'à l'identification et l'évaluation des indicateurs de bonne production.

\section{Enquête pisciculteurs}

Une enquête a été réalisée en Côte d'Ivoire en 2013 auprès de 301 pisciculteurs, précisément dans quinze régions à vocation piscicole. Elle a porté sur la gestion des fermes, les stratégies alimentaires et les données de production. La gestion de la ferme est relative à la qualification du gestionnaire, aux bonnes pratiques aquacoles et à l'encadrement des fermes. Les stratégies alimentaires sont relatives aux systèmes d'élevage pratiqués, à la qualité de l'aliment utilisé et aux pratiques alimentaires lorsque la production piscicole relate les données de croissance et de production. Le Tableau 1 présente les composantes associées aux thématiques définies.

L'enquête a été réalisée à l'aide d'un questionnaire élaboré avec le logiciel Sphinx 4.5 et des fiches de renseignements élaborées à partir du logiciel Word. Les fermes enquêtées ont été visitées puis les documents de gestion ont été consultés. Le choix des systèmes semi intensif ou extensif de pisciculture a été fait selon les descriptions de Lacroix (2004) et Lazard (2009). Concernant le cycle de production, les différentes étapes pratiquées par les pisciculteurs ont permis d'effectuer un classement en 4 cycles. Le cycle normal caractérisé par un cycle d'élevage complet, le cycle unique s'effectuant sur une population non sexée; dans ce cas, les structures de reproduction sont en même temps les milieux de croissance, le cycle «reproduction + grossissement », caractérisé par le sexage du tilapia après la reproduction suivie de l'élevage dans d'autres structures jusqu'à la taille marchande et le cycle de « reproduction + pré grossissement + grossissement », dans lequel l'on procède à une autre redistribution des poissons après sexage, avec réduction de la densité de mise en charge pour la phase du grossissement. Les informations relevées sur les fermes ont été traitées à partir du logiciel Sphinx 4.5 et présentées sous forme de pourcentage.

\section{Identification et évaluation des indicateurs}

$\mathrm{Au}$ total, 16 indicateurs ont été sélectionnés et évalués. Il s’agit de sept indicateurs de gestion de fermes, cinq indicateurs de l'alimentation des poissons et quatre indicateurs de production. Le Tableau 2 présente les indicateurs sélectionnés et leur code d'identification. Ceux-ci seront évalués dans les différents systèmes inventoriés.

\section{RESULTATS}

\section{Systèmes piscicoles pratiqués}

Quatre différents systèmes de production ont été identifiés sur les fermes visitées. $\mathrm{Ce}$ sont les systèmes intensif $(1,33 \%)$, semi intensif $(51,83 \%)$ et extensif $(42,86 \%)$ pratiqués en pisciculture simple. La rizipisciculture $(3,99 \%)$ est le mode de pisciculture intégré identifié sur les fermes.

Le système intensif est caractérisé par l'élevage en monoculture de silure et/ou de tilapia dans les étangs, les bacs en béton ou en aluminium et les cages flottantes. Dans ce système, les poissons sont nourris exclusivement avec les aliments industriels commerciaux importés ou locaux de manière intensive. Le cycle normal de production et le «prégrossissement + grossissement» sont pratiqués. 
Le système semi intensif est caractérisé par l'élevage en mono ou polyculture de tilapia, de silure, de machoiron, de carpe, d'heterotis et de parachana. La croissance a lieu dans les étangs, les cages enclos, les happas, les bassins en ciment et les étangs barrage en mono ou en polyculture. Les poissons sont nourris régulièrement avec les sous produits agro-alimentaires, les aliments fabriqués par les pisciculteurs eux-mêmes et les aliments industriels commerciaux locaux. Les cycles d'élevage pratiqués sont le cycle normal, le cycle de reproduction + pregrossissement, et le cycle de reproduction + pregrossissement + grossissement.

En système extensif, le tilapia, les silures, la carpe, l'heterotis, et le parachana sont élevés en étangs, en étangs barrage, et en barrage en monoculture ou en polyculture. Les poissons sont nourris occasionnellement avec les sous produits agro-alimentaires et les aliments non conventionnels.

Dans la rizipisciculture, le tilapia et l'hétérothis sont les espèces de poissons élevés en étangs ou en étang de barrage en monoculture ou en polyculture. Les sous produits sont distribués aux poissons de façon régulière ou occasionnelle. Le cycle unique de production ou le grossissement des poissons sont effectués dans ce système.

Le schéma récapitulatif des systèmes identifiés est présenté à la Figure 1.

Evaluation des indicateurs de gestion des fermes par système piscicole

Le profil de gestion des fermes par système d'élevage présenté à la Figure 2 a permis d'observer que la gestion des fermes varie d'un système à un autre. Le système intensif est caractérisé par $100 \%$ de pratique des sept indicateurs de gestion évalués. Le système semi intensif est caractérisé par plus de $50 \%$ des fermes possédant un cahier de charge, effectuant les pêches de contrôle, le trie et le sexage des poissons de taille inférieur à $40 \mathrm{~g}$. Le système extensif se distingue par un taux de suivi inférieur à $50 \%$ par rapport à l'ensemble des indicateurs de gestion évalués tandis qu'en rizipisciculture, plus de $50 \%$ des pisciculteurs effectuent des tris et sexent le tilapia à la taille inférieure à $40 \mathrm{~g}$.

\section{Evaluation des indicateurs de l'alimentation des fermes par système piscicole}

Les indicateurs de l'alimentation évalués dans les différents systèmes sont présentés à la Figure 3. Les résultats permettent d'observer que dans le système intensif de pisciculture, $100 \%$ de pisciculteurs utilisent l'aliment commercial, une table de rationnement pour quantifier l'aliment à distribuer et une intensité de nourrissages supérieurs à $100 \mathrm{~kg} / \mathrm{ha} / \mathrm{an}$. Aussi, plus de 50\% des pisciculteurs utilisent un type d'aliment par espèce élevée. Au sein du système semi intensif, plus de $50 \%$ des pisciculteurs utilisent un aliment commercial. Par ailleurs, le mode d'élevage extensif et la rizipisciculture sont caractérisés par un très faible taux d'expression de tous les indicateurs de l'alimentation évalués.

\section{Evaluation des indicateurs de la productivité des fermes par système piscicole}

Les différents taux d'expression des indicateurs de production évalués par systèmes piscicoles sont présentés à la Figure 4. Le système intensif est caractérisé par un taux d'expression de $100 \%$ des pisciculteurs ayant une durée de production inférieure à 8,5 mois et le rendement supérieur à 10000 $\mathrm{kg} / \mathrm{ha} / \mathrm{an}$. Aussi, plus de $50 \%$ des pisciculteurs en intensif ont-ils un GMQ tilapia supérieur à $1,5 \mathrm{~g} / \mathrm{j}$. Le semi intensif est caractérisé par un taux d'expression de tous les indicateurs de production inférieurs à 50\%. L'extensif et la rizipisciculture présentent une très faible expression des indicateurs de production. 
Tableau 1: Thématiques, composantes et paramètres recommandés (Craig et Helfrich, 2002; Lacroix, 2004 ; Brechbühl, 2009; Lazard, 2009).

\begin{tabular}{|c|c|c|}
\hline Thématiques & Composantes & Paramètres recommandés \\
\hline $\begin{array}{l}\text { Gestion de la } \\
\text { ferme }\end{array}$ & $\begin{array}{l}\text { - Qualification du } \\
\text { gestionnaire } \\
\text { - Bonnes pratiques } \\
\text { aquacoles } \\
\text { - Encadrement }\end{array}$ & $\begin{array}{c}\text { - Gestionnaire qualifié en pisciculture } \\
\text { - Enregistrement des données d'élevage } \\
\text { - Pêches de contrôle mensuel } \\
\text { - Tri des poissons par stade de développement } \\
\text { - Sexage du tilapia à la taille } \leq 40 \mathrm{~g} \\
\text { - Cycle normal de production } \\
\text { - Encadrement par un spécialiste }\end{array}$ \\
\hline $\begin{array}{l}\text { Stratégie } \\
\text { d'alimentation }\end{array}$ & $\begin{array}{l}\text { - Système d'élevage } \\
\text { - Qualité de l'aliment } \\
\text { - Pratiques alimentaires }\end{array}$ & $\begin{array}{c}\text { - Système intensif } \\
\text { - Système semi intensif } \\
\text { - Aliment de qualité } \\
\text {-Tableau de rationnement pour quantifier l'aliment } \\
\text {-Utilisation d'un aliment par stade de croissance } \\
\text { - Utilisation d'un aliment par espèce } \\
\text { - Intensité de nourrissage } \geq 100 \mathrm{~kg} / \mathrm{ha} / \mathrm{j}\end{array}$ \\
\hline Production & Paramètres de productions & $\begin{array}{l}\text { - Durée de production de poissons marchands } \leq 8 \\
\text { mois } \\
\text { - GMQ tilapia } \geq 1,5 \mathrm{~g} / \mathrm{j} \\
\text { - Poids marchand tilapia } \geq 350 \mathrm{~g} \\
\text { - Rendement } \geq 10000 \mathrm{~kg} / \mathrm{ha} / \mathrm{an}\end{array}$ \\
\hline
\end{tabular}

GMQ: gain moyen quotidien

Tableau 2 : Codification des indicateurs identifiés.

\begin{tabular}{lcl}
\hline Thématiques & $\begin{array}{c}\text { Numéro des } \\
\text { indicateurs }\end{array}$ & \multicolumn{1}{c}{ Indicateurs $(\%)$} \\
\hline \multirow{4}{*}{ Gestion de la ferme } & IG-1 & Gestionnaire qualifié en pisciculture \\
& IG-2 & Pisciculteur dotée d'un cahier de charge \\
& IG-3 & Pisciculteur effectue les pêches de contrôle \\
& IG-4 & Pisciculteur tri les poissons \\
& IG-5 & Pisciculteur sexe le tilapia à la taille $\leq 40 \mathrm{~g}$ \\
& IG-6 & Pisciculteur pratique le cycle normal de production \\
& IG-7 & Pisciculteur est suivi par un spécialiste \\
\hline \multirow{4}{*}{ Alimentation } & IA-1 & Pisciculteur utilise un aliment commercial \\
& IA-2 & Pisciculteur utilise une table de rationnement \\
& IA-3 & Pisciculteur utilise un aliment par stade de développement \\
& IA-4 & Pisciculteur utilise un aliment par espèce \\
& IA-5 & Intensité de nourrissage $\geq 100 \mathrm{~kg} / \mathrm{ha} / \mathrm{j}$ \\
\hline \multirow{4}{*}{ Données de production } & IP-1 & Durée de production de poisson marchands $\leq 8,5$ mois \\
& IP-2 & GMQ tilapia $\geq 1,5 \mathrm{~g} / \mathrm{j}$ \\
& IP-3 & Poids marchand du tilapia $\geq 350 \mathrm{~g}$ \\
& IP-4 & Rendement de la ferme $\geq 10000 \mathrm{~kg} / \mathrm{ha} / \mathrm{an}$ \\
\hline
\end{tabular}


H. A. YAO et al. / Int. J. Biol. Chem. Sci. 10(3): 1086-1097, 2016

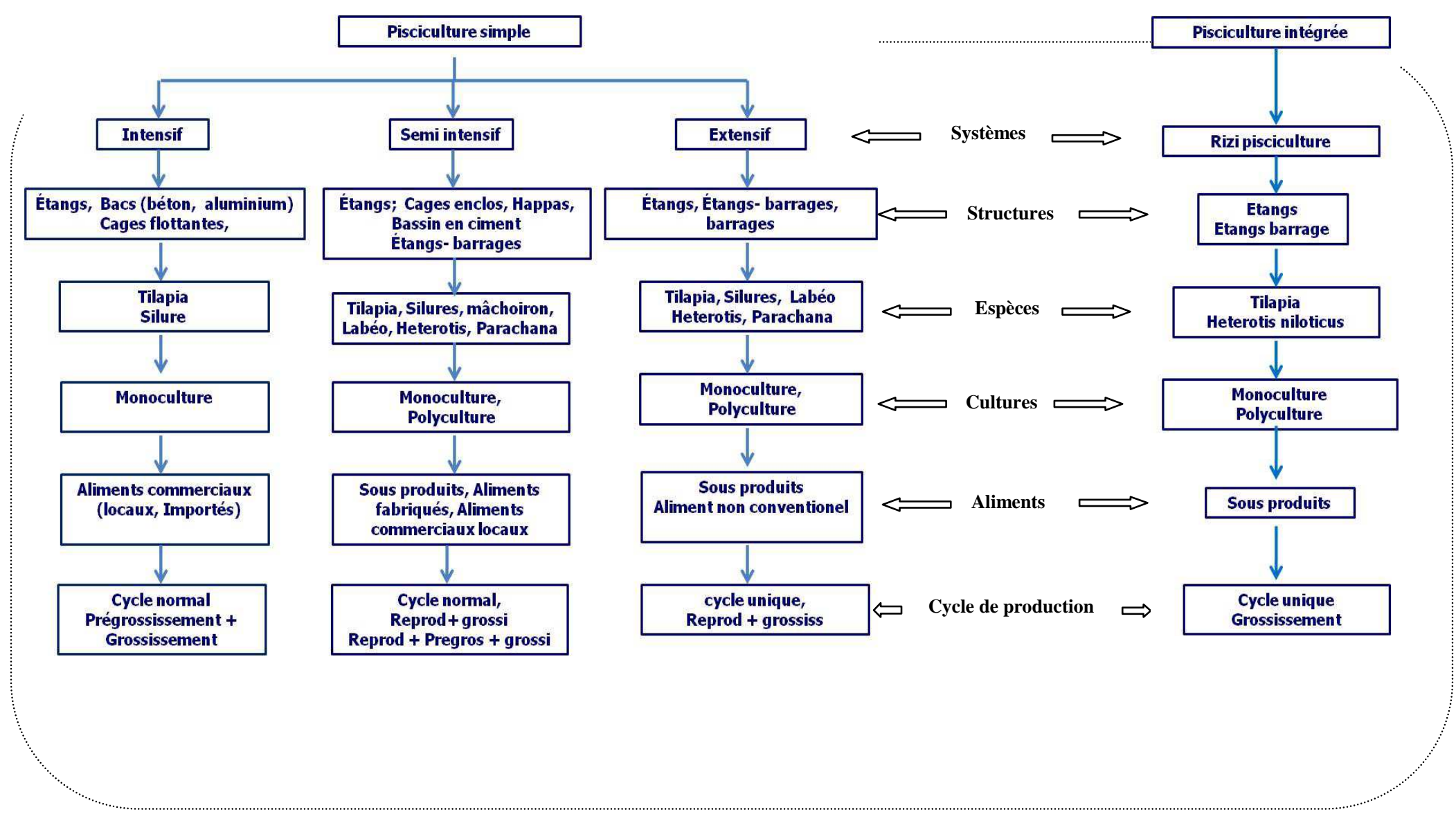

Figure 1 : Schéma récapitulatif des différents systèmes piscicoles pratiqués. 

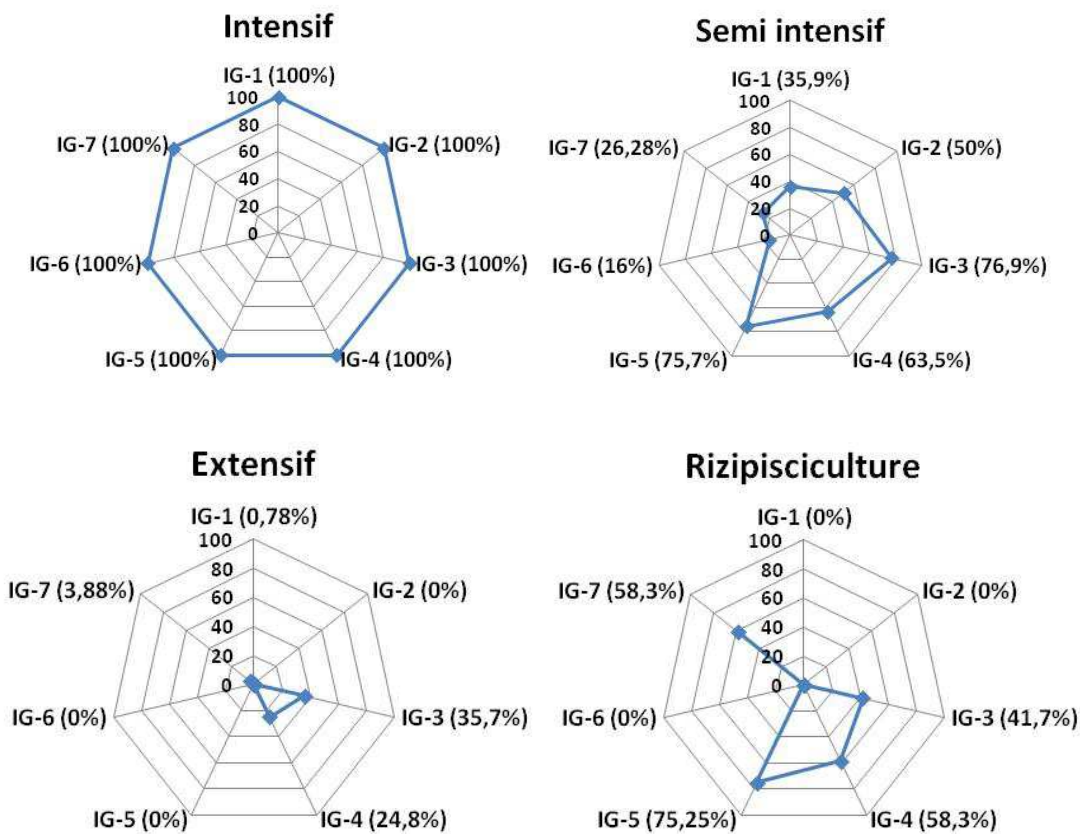

Figure 2: Profil de gestion par système piscicole. IG-1: Gestionnaire qualifié en pisciculture; IG-2 : Pisciculteur doté d'un cahier de charge ; IG-3 : Pisciculteur effectue les pêches de contrôle ; IG-4 : Pisciculteur trie les poissons ; IG-5 : Pisciculteur sexe le tilapia à la taille $\leq 40 \mathrm{~g}$; IG-6 : Pisciculteur pratique le cycle normal de production ; IG7 : Pisciculteur est suivi par un spécialiste.
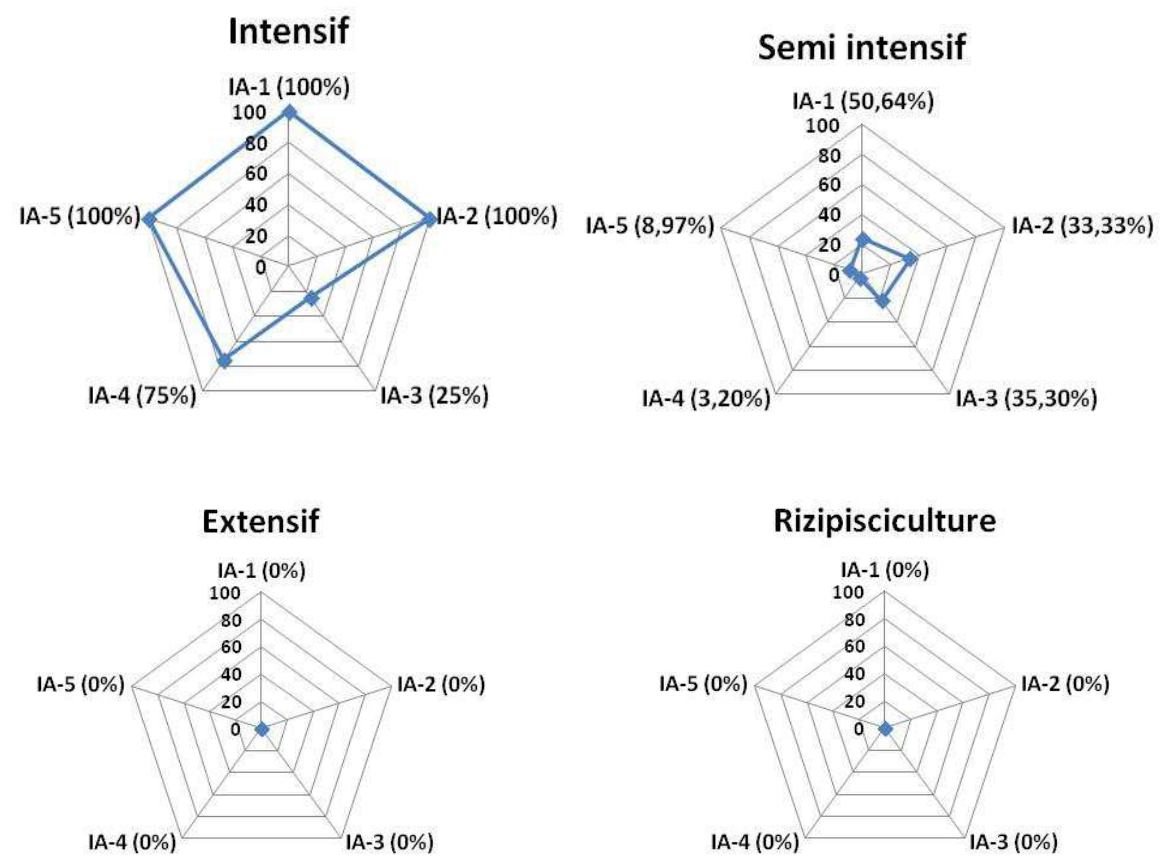

Figure 3 : Profil d'alimentation par système piscicole. IA-1 : Pisciculteur utilise un aliment commercial ; IA2: Pisciculteur utilise un tableau de rationnement; IA-3: Pisciculteur utilise un aliment par stade de développement; IA4 : Pisciculteur utilise un aliment par espèce ; IA-5 : Intensité de nourrissage $\geq 100 \mathrm{~kg} / \mathrm{ha} / \mathrm{j}$. 


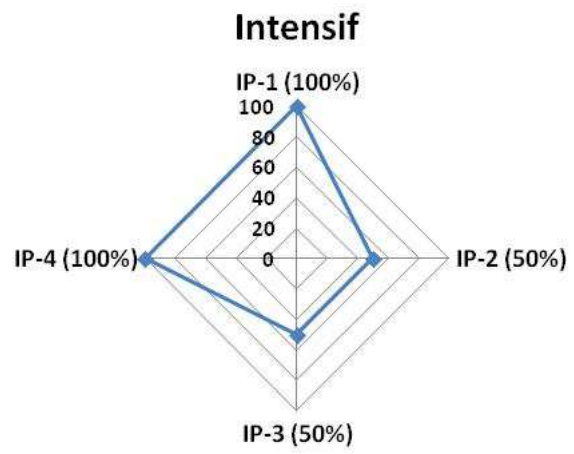

Extensif

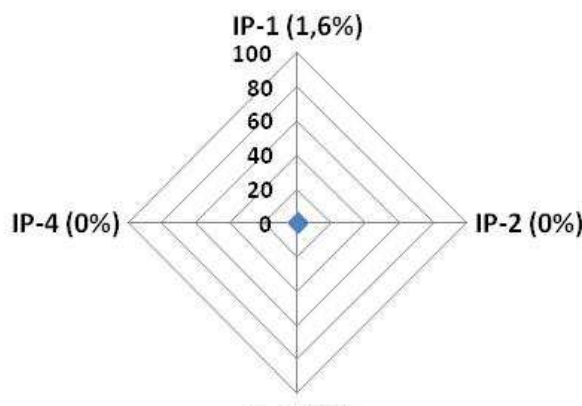

IP-3 (0\%)

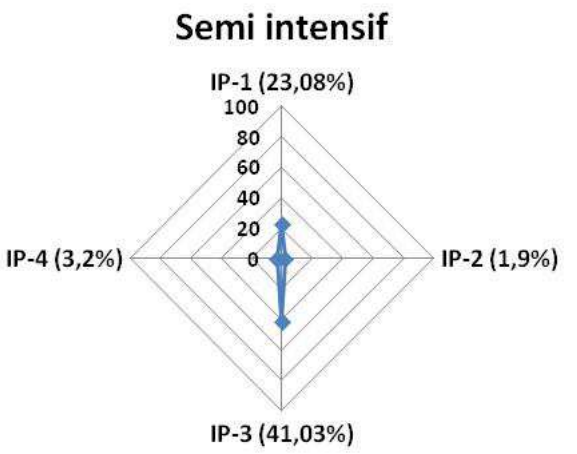

Rizipisciculture

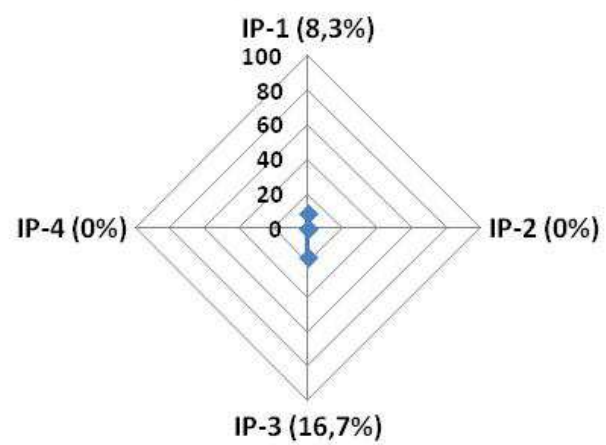

Figure 4 : Profil de production par système piscicole. IP-1 : Durée de production de poissons marchands $\leq$ à 8,5 mois; IP-2: GMQ tilapia $\geq 1,5 \mathrm{~g} / \mathrm{j}$; IP-3 : Poids marchand tilapia $\geq 350 \mathrm{~g}$; IP-4 : Rendement de la ferme $\geq 10000$ $\mathrm{kg} / \mathrm{ha} / \mathrm{an}$.

\section{DISCUSSION}

Les systèmes d'élevage semi intensif et extensif sont les plus rencontrés sur les fermes piscicoles enquêtées. Ce constat a également été rapporté dans la plupart des pays de l'Afrique de l'Ouest (Hecth, 2007; FAO, 2014a). En effet, la production aquacole de ces pays est majoritairement issue de petites superficies en eaux appartenant à des petits opérateurs ruraux considérant la pisciculture comme une activité secondaire (Gabriel et al., 2007). La faible fréquence du système intensif serait due à son haut niveau d'investissement et de technicité, à l'intensification de l'activité aquacole par le suivi et le contrôle régulier et permanent de l'aliment, de la qualité de l'eau et de la production, nécessitant d'important investissement (Lazard, 2009; Ozigo et al., 2014). De ce fait, la pratique de ce système est majoritairement réservée aux gros investisseurs et entreprises privées (FAO, 2014a). Par ailleurs, la pratique de la rizipisciculture est récente en Côte d'Ivoire par rapport aux autres systèmes d'élevage. Sa faible fréquence pourrait s'expliquer par la culture concomitante de riz et de poissons nécessitant la maîtrise de ces deux cultures (Avit et al., 2012). De ce fait, elle est pratiquée en majorité dans la région du Haut Sassandra et reste très peu répandue sur tout le territoire ivoirien (Kaudjis, 2009).

$\mathrm{La}$ présente investigation permet d'observer dans le système intensif un bon niveau de gestion, de suivi et le respect de la majorité des pratiques alimentaires conduisant à une faible durée de production de poissons marchands et à des rendements piscicoles élevés chez tous les pisciculteurs. Cependant, les gains moyens quotidiens et les poids marchands de tilapia devraient être améliorés sur 50\% de ces fermes. Les faibles croissances de ces fermes pourraient être dues à la non 
utilisation d'aliments de qualité spécifiques aux stades de développement et aux espèces de poissons élevés par 25 à $50 \%$ des pisciculteurs en système intensif. En effet, Koumi et al. (2015) rapportent que seuls les aliments commerciaux importés coûteux sont adaptés aux différents stades de croissances des poissons élevés. Les pisciculteurs en système d'élevage intensif utilisant les aliments commerciaux locaux se trouvent donc limités par l'absence d'aliment de qualité. Ce qui pourrait expliquer les valeurs de gain de poids tilapia inférieur à $1,5 \mathrm{~g} /$ jour chez $50 \%$ des pisciculteurs en système intensif. En effet, l'aliment représente un intrant majeur pour la croissance et la santé des poissons dans ce système (Craig et Helfrich, 2002).

Le système semi intensif en Côte d'Ivoire contribue très peu à la production de poissons marchands compétitifs en quantité suffisante, du fait du faible niveau de technicité, de la rareté des gestionnaires qualifiés et l'absence d'assistance technique. De plus, le non-respect du cycle normal de production et des stratégies d'alimentation par la majorité de ces fermes contribue à des rendements annuels de plus de 10000 $\mathrm{kg} / \mathrm{ha} / \mathrm{an}$ chez seulement $3,2 \%$ des pisciculteurs. En effet, selon plusieurs auteurs, les rendements en système semi intensif varient entre 1,5 et 15 tonnes/ha/an (Lazard, 2009 ; Mikosalek et al., 2009 ; Lacroix, 2004). Les valeurs de production enregistrées dans notre étude en majorité inférieures à 10000 $\mathrm{kg} / \mathrm{ha} / \mathrm{an}$ révèlent d'un rendement minimal sur ces fermes. Il conviendrait de définir de meilleures conditions de gestion de suivi et d'alimentation des fermes en système semi intensif afin de maximiser leur production. En effet, ce système est préférentiellement utilisé et est très productif dans plusieurs pays à forte production aquacole tel que l'Amérique l'Asie et l'Afrique en particulier l'Egypte, le Nigéria, le Ghana, l'Ouganda et la Zambie (Lazard, 2009). Aussi, la pratique de la fertilisation (organique et/ou inorganique) très peu observée sur les fermes visitées, augmente considérablement la production d'aliments naturels dans les étangs et améliore la croissance des poissons et le rendement à moindre coûts (Ayinla et al., 2007; Agadjihouede et al., 2011; Ozigo et al., 2014). La fertilisation des étangs, très peu observée sur les fermes visitées associée au nourrissage régulier des poissons pourrait considérablement améliorer la production piscicole en système semi intensif.

Les systèmes extensif et la rizipisciculture sont en général caractérisés par une faible technicité et un faible rendement $(0,5$ à 2 tonnes/ha/an) à cause d'une production basée sur la productivité naturelle de l'environnement (Lacroix, 2004 ; Lazard, 2009). Cependant, les meilleures croissances des poissons élevés en rizipisciculture par rapport à l'extensif rapportées dans cette étude pourraient s'expliquer par une plus forte disponibilité de l'aliment naturel dans le milieu. Celle-ci est favorisée par les intrants destinés à la culture du riz, la présence du periphyton, des produits issus de la décomposition des végétaux et des insectes sur de petites surfaces utilisées améliorant ainsi la croissance des poissons (Avit et al., 2012; Fermon, 2013). Par ailleurs, le recours au système extensif dans le monde à beaucoup diminué et est passé de $50 \%$ à 33,3\% du fait de l'intensification des systèmes de production entamée depuis les années 1990 (Li, 2002). Hecht (2007) rapporte également un développement notable des fermes intensives à caractère commercial au détriment des systèmes extensifs et non commercial depuis les années 2000 en Uganda, au Kenya, en Malawi, au Cameroun, au Nigéria et au Ghana. Cette conversion a contribué à une augmentation considérable de la production piscicole de ces pays. A l'instar de ceux-ci, une politique visant à réduire la proportion du système extensif et à réorienter la pisciculture peut augmenter la production piscicole ivoirienne. 


\section{Conclusion}

La pisciculture en Côte d'Ivoire est dominée par la pratique du système extensif très peu productif. Le système intensif subit une faible accessibilité d'aliments commerciaux locaux spécifiques aux différents stades de développement des poissons et le semi intensif, un niveau de gestion rudimentaire et des stratégies alimentaires non adaptées. L'amélioration de la productivité des fermes piscicoles nécessite le développement d'une industrie d'aliment de poisson en Côte d'Ivoire et la définition d'une stratégie de suivi, d'accompagnement et d'intensification de l'activité piscicole.

\section{CONFLIT D'INTERETS}

Les auteurs déclarent qu'il n'y a aucun conflit d'intérêt.

\section{CONTRIBUTIONS DES AUTEURS}

HAY, RAK, CSKN et CBA ont participés aux enquêtes sur les fermes, à la collecte et au traitement des données. HAY, R $\mathrm{AK}$ et CSKN ont effectués la rédaction de l'article sous les directives de CBA et de PEK qui ont également révisés l'article avant soumission.

\section{REMERCIEMENTS}

Les auteurs remercient le Programme d'Appui Stratégique à la Recherche Scientifique (PASRES) pour leur soutien. Les auteurs expriment également leur sincères remerciements aux différentes structures étatiques, aux associations de pisciculteurs et toutes les personnes qui ont facilité la réalisation de cette étude.

\section{REFERENCES}

Agadjihouede H, Montchowui E, Chikou A, Laleye PA. 2011. Libération comparée de sels dans l'eau par la minéralisation de l'azolla, la bouse de vache, la fiente de volaille et les sons de riz et de maïs utilisés en pisciculture. Int. J. Biol.
Chem. Sci., 5(5): 1883-1897. DOI : http://dx.doi.org/10.4314/ijbcs.v5i5.10

Avit J-BLF, Boni KY, Kouassi NC, Konan KKF, Assemian O, Alloukou JR. 2012. Conditions écologiques de production de fingerlings de Oreochromis niloticus (Linné, 1758) en association avec le riz Wita 12 en étang. J. Appl. Biosci., 59 : 4271-4285.

DOI: http://www.m.elewa.org/JABS/2012/59/ 1.pdf

Ayinla OA. 2007. Analysis of feeds and fertilizers for sustainable aquaculture development in Nigeria. In Study and Analysis of Feeds and Fertilizers for Sustainable Aquaculture Development, Hasan MR, Hecht T, De Silva SS, Tacon AGJ (eds). FAO Fisheries Technical Paper: Rome; 453- 470.

Brechbühl A. 2009. The future of pisciculture in southern Côte d'Ivoire. Bachelor thesis, Federal Institute of Technology Agri-food and Agri-environmental Economics Group, Zürich (Swiss), p. 50. Craig S, Helfrich A. 2002. Understanding fish Nutrition, Feeds and Feeding. Virginia Cooperation Extension: Virginia.

Elegbe HA, Toko II, Agbohessi P, Blé MC, Banag A, Chikou A, Tomedi ME, Laleye P. 2015. Co-culture Clarias gariepinus-Oreochromis niloticus : quels avantages pour l'amélioration des performances zootechniques et économiques des poissons élevés dans les « whedos» du delta de l'Ouémé au Bénin ? Int. J. Biol. Chem. Sci., 9(4): 1937-1949.

DOI: http://dx.doi.org/10.4314/ijbcs.v9i4.19

FAO. 2011. The State of World Fisheries and Aquaculture. FAO: Rome.

FAO. 2013. Le Rôle de l'Aquaculture dans l'Amélioration de la Nutrition: Possibilités et Défis. FAO COFI ; AQ/VII/2013/7 : Saint Petersburg.

FAO. 2014a. La Situation Mondiale des Pêches et de l'Aquaculture. Possibilité et défis. FAO : Rome. 
FAO. 2014b. Fisheries and Aquaculture Information and Statistics Service. FAO: Rome.

http://www.fao.org/figis/servlet/SQServlet $? \mathrm{ds}=$ Aquaculture $\& \mathrm{k} 1=\mathrm{COUNTRY} \& \mathrm{k} 1 \mathrm{v}=$ $1 \& \mathrm{k} 1 \mathrm{~s}=107 \&$ outtype $=\mathrm{html}$

Fermon Y. 2013. La Pisciculture de Subsistance en Etang en Afrique Subsaharienne. Manuel Technique, ACF - International Network: Paris.

Gabriel UU, Akinrotimi OA, Bekibele DO, Onunkwo DN, Anyanwu PE. 2007. Locally produced fish feed: potentials for aquaculture development in subSaharan Africa. Afr. J. Agric. Res., 2: 287-295.

DOI: http://www.academicjournals.org/app/w ebroot/article/article1380787639_Gabrie $1 \% 20$ et\%20al..pdf

Gobert H. 2008. Suivi évaluation de la durabilité de la pisciculture d'un territoire; Application des indicateurs de durabilité co-construits par les parties prenantes de la pisciculture de la Province de l'Ouest Cameroun. Mémoire de fin d'études pour l'obtention du Diplôme d'Agronomie Approfondie (DAA) spécialisation halieutique, Agro Campus OuestCIRAD, Rennes (France), p. 60.

Harpaz S. 2007. Catfish nutrition-aspects to consider. In Proceeding of a Workshop on the Development of a Genetic Improvement Program for African Catfish Clarias gariepinus, RW Ponzoni, Nguyen HN (eds). World Fish Center: Accra; 79-81.

Hecht T. 2007. Review of feeds and fertilizers for sustainable aquaculture development in sub-Saharan Africa. In Study and Analysis of Feeds and Fertilizers for Sustainable Aquaculture Development, Hasan MR Hecht T, De Silva SS, Tacon AGJ (eds). FAO Fisheries Technical paper: Rome; 453- 470.

Hishamunda N, Cai J, Leung P. 2011. Aquaculture Commerciale et Croissance
Economique, Réduction de la Pauvreté et Sécurité Alimentaire: Cadre d'Evaluation. FAO Document technique sur les pêches et l'aquaculture: Rome.

Hossain MA. 2011. Fish as source of n-3 polyunsaturated fatty acids (PUFAs), which one is better-farmed or wild. $a d v$. J. Food Sci. Technol., 3: 455-466. DOI : http://citeseerx.ist.psu.edu/viewdoc/dow nload?doi=10.1.1.461.1420\&rep=rep1\&t ype $=$ pdf

Khaudjis AJ. 2009. Evaluation des Règlementations et des Programmes Aquacoles : Côte d'Ivoire. SARNISSA : Bouaké.

Koumi AR, Kimou BN, Atse BC, Ouattara IN, Kouame LP. 2015. Fish feeds used in Côte d'Ivoire: Nature, quality, use and productivity. Asian J. Agric. Food Sci., 3: $\quad 225-236 . \quad$ DOI : http://www.ajouronline.com/index.php? journal=AJAFS\&page $=$ article $\& o p=v i e$ w\&path $\% 5 B \% 5 \mathrm{D}=2542$

Mikosaleck O, Barlet B, Chia E, Pouomogne V, Tomedi ETM. 2009. Develloppement de la petite pisciculture marchande au Cameroun: la recherche-action en partenariat. Cah. Agric., 18: 270-276. DOI:

http://publications.cirad.fr/une_notice.ph $\mathrm{p} ? \mathrm{dk}=549820$

Munguti JM, Musa S, Orina PS, Kyule DN, Opiyo MA, Charo-Karisa H, Ogello EO. 2014. An overview of current status of Kenyan fish feed industry and feed management practices, challenges and opportunities. Int. J. Fish. Aquatic Studies, 1(6): 128-137.

Lacroix E. 2004. Pisciculture en Zone Tropicale. GTZ, GFA Terra Systems: Hamburg.

Lazard J. 2009. La pisciculture des tilapias. Cah. Agric., 18: 174-182, pisciculture: le poisson de demain, Synthèse, p. 24. DOI :

http://www.jle.com/fr/revues/agr/e- 
docs/la_pisciculture_des_tilapias_28147

6/article.phtml?tab=texte

Li SF. 2002. Aquaculture research and its relation to development in China. In Agricultural Development and the Opportunities for Aquatic Resources Research in China, Zhang LX, Liu J, Li SF, Yang NS, Gardiner PR (eds). WorldFish Center: Penang; 17-28.

OCDE-FAO. 2011. Produits de la pêche et de l'aquaculture. In Perspectives Agricoles de l'OCDE et de la FAO 2011-2020, OCDE/FAO (ed). OCDE/FAO: Rome; 171-184.

Ozigbo E, Anyadike C, Adegbite O, Kolawole P. 2014. Review of aquaculture production and management in Nigeria. American J. Experimental Agric., 40: 1137-1151.

Ranjet K, Kurup BM. 2013. Economic analysis of polder based freshwater prawn farming systems in Kuttanad, India. Int. J. Fish. Aquaculture, 5: 110-
$121 \quad$ DOI: http://www. academicjournals.org/journal/IJFA/articl e-full-text-pdf/4AE7B859381

Toko II, Attakpa EY, Baco MN, Gouda A-I. 2011. Analyse des systèmes piscicoles dans la Vallée du Niger (Nord Bénin). Int. J. Biol. Chem. Sci., 5(5): 1993-2003.

Toko II, Bachabi SF-X, Houndji AMB, Fiogbe ED, Kestemont P. 2013. Water quality and productivity of the traditional aquaculture system (whedos) in Oueme delta (Benin). Int. J. Biol. Chem. Sci., 7(3): 1298-1312. DOI : http://dx.doi.org/10.4314/ijbcs.v7i3.35

Toppe J, Bondad-Reantaso MG, Hasan MR, Josupeit H, Subasinghe RP, Halwart M, James D. 2012. Aquatic biodiversity for sustainable diets: The role of aquatic foods in food and nutrition security. In Sustainable Diets and Biodiversity, FAO (ed). FAO: Rome; 94-101. 